11,01

\title{
Калориметрия процессов кристаллизации двухкомпонентных сплавов
}

\author{
(C) Л.В. Спивак ${ }^{1}$, Н.Е. Щепина ${ }^{2}$ \\ ${ }^{1}$ Пермский государственный национальный исследовательский университет, \\ Пермь, Россия \\ ${ }^{2}$ Естественно-научный институт Пермского государственного национального исследовательского университета, \\ Пермь, Россия \\ E-mail: Ispivak2@mail.ru, neshchepina@mail.ru
}

(Поступила в Редакцию 1 февраля 2018 г.)

С использованием дифференциальной сканирующей калориметрии высокого разрешения исследованы процессы кристаллизации в чистых металлах и в некоторых двухкомпонентных сплавах на их основе. При кристаллизации обнаружены скачки теплового потока по достижению линий ликвидуса. Предполагается, что эти явления связаны с образованием в жидкости большого количества локальных микрообъемов (концентрационных флуктуаций), обогащенных ведущим кристаллизацию компонентом. Их появление предшествует началу процессов спонтанного образования и роста кристаллов новой фазы.

DOI: 10.21883/FTT.2018.12.46733.029

\section{1. Введение}

Теоретический анализ процессов кристаллизации восходит к работам Курдюмова, Мейла, Миркина [1,2], описывающих кинетику этого процесса. В дальнейшем анализ базировался на идеях Гиббса, позволивших записать уравнения для скорости образования центров кристаллизации и их дальнейшего роста [1-3]. Как правило, при этом рассматривалась кристаллизация однокомпонентных жидкостей. Для двухкомпонентных жидкостей кристаллизация сплавов не эвтектического состава не нашла достаточного развития в теоретических исследованиях, если не считать отдельных работ, описание которых представлено в различных обзорах (см., например, [4-5]). Экспериментальные исследования представлены в основном классическими работами Таммана [3,4] и группы Давиденкова [1], которые были проведены при кристаллизации органических жидкостей, а Торнбаллом - на металлах [3,4].

Несомненно, большие достижения связаны с экспериментами по построению диаграмм состояния металлических сплавов, позволяющих определить области существования отдельных фаз или структурных составляющих. Начиная с работ Курнакова [6], при исследовании фазовых превращений широко используются физические методы наблюдения за процессами кристаллизации или распада пересыщенных твердых растворов. Среди них наиболее часто используются методы дифференциального термического анализа (DTA), позволяющие в некоторых случаях достаточно точно определять начало и завершение структурно-фазовых превращений. Однако такие исследования не дают информацию о процессах, проходящих в межкритическом интервале температур.

Теоретические подходы к описанию процессов затвердевания, зарождения и роста кристаллов новой фазы оперируют представлениями об изменении свободной энергии системы при переходе из жидкого состояния в кристаллическое. Однако, экспериментальных исследований, направленных на изучение характера калориметрических эффектов при кристаллизации двух компонентных сплавов, относительно невелико.

В последнее десятилетие появились методики и аппаратура для дифференциальной сканирующей калориметрии (DSC) высокого разрешения, позволившие вновь вернуться к экспериментальным исследованиям в этом направлении. Тем не менее, основное внимание при этом было уделено исследованию кристаллизации аморфных металлических сплавов, представляющих в большинстве случаев многокомпонентные системы [7-9]. Существенно меньше работ по DSC процессов кристаллизации расплавов. Но и в этом случае рассматривалась кристаллизация многокомпонентных сплавов сугубо промышленного назначения, и вопросы технологии носили в них приоритетный характер (см. например, [10,11]). Одним из исключений можно считать работу [12], в которой проведен DSC анализ кристаллизации доэвтектических, эвтектических и заэвтектических сплавов системы Al-Si. Но и здесь основной упор был сделан на изучение влияния модификаторов на тепловые эффекты при кристаллизации и измельчении структуры сплавов.

В настоящей работе впервые рассмотрено применение этих методик для таких простых систем, общий тип которых можно представить диаграммой состояния II рода, например, системы $\mathrm{Bi}-\mathrm{Sn}$. Для таких систем проведена дифференциальная сканирующая калориметрия сплавов с относительно низким содержанием одного из компонентов и не претерпевающих при охлаждении эвтектической реакции (I тип). II, III и IV типы относятся к сплавам доэвтектического, эвтектического и заэвтектического состава. 


\section{2. Методика}

Объектом исследования были выбраны чистые металлы ( $\mathrm{Sn}, \mathrm{Bi}, \mathrm{Pb}, \mathrm{Zn}, \mathrm{Al}, \mathrm{Ag}, \mathrm{Au}, \mathrm{Cu})$ и двухкомпонентные сплавы систем $\mathrm{Pb}-\mathrm{Sn}, \mathrm{Pb}-\mathrm{Bi}, \mathrm{Bi}-\mathrm{Sn}, \mathrm{Al}-\mathrm{Cu}$, представленных дюралями Д1 $(4.1 \% \mathrm{Cu}, 0.7 \% \mathrm{Mg}, 0.8 \% \mathrm{Mn})$ и Д19 (4.0\% Cu, 1.7\% Mg, 0.5\% Mn).

Данные по дифференциальной сканирующей калориметрии были получены с помощью прибора STA „Jupiter“ 449 фирмы Netzsch. Нагрев и охлаждение производили со скоростью 5 и $10 \mathrm{~K} / \mathrm{min}$ в атмосфере аргона (99.999\% Ar). Скорость потока газа 25-30 ml/min. Обработка экспериментальных данных по DSC реализована с использованием программного обеспечения „Proteus Analyses“" и пакета „Fityk“.

Для исключения возможности появления при исследовании сплавов артефактов, обусловленных особенностями эксперимента, проводили сглаживание экспериментальных данных полиномом восьмой степени. Этот же прием использовался и при анализе данных по первой (DDSC) и второй (DDDSC) производных сигнала DSC по температуре. В экспериментах с чистым алюминием (99,999\% вес. Al) показано, что температурные интервалы и тепловой эффект превращения, определенные до сглаживания экспериментальных кривых и после их аппроксимации, практически одинаковы. Расхождение составляло около $1 \%$. Приведенные в сообщении результаты относятся к скорости охлаждения $10 \mathrm{~K} / \mathrm{min}$.

\section{3. Экспериментальные результаты и их обсуждение}

На рис. 1. показана DSC кривая при охлаждении сплава эвтектического состава и его вторая производная по температуре. Внешне обе зависимости весьма похожи на поведение DSC и DDDSC при кристаллизации однокомпонентного сплава (см. рис. 2). Это не удивительно, поскольку эвтектическая реакция характеризуется

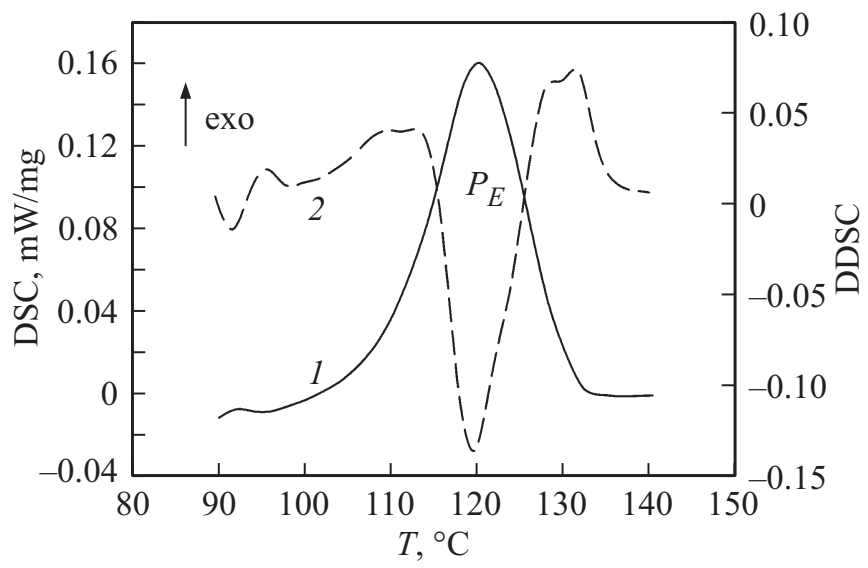

Рис. 1. Зависимость сигнала DSC (1) и DDDSC (2) при образовании эвтектики $\mathrm{Bi}-\mathrm{Sn}(55 \%$ вес. $\mathrm{Sn})$.

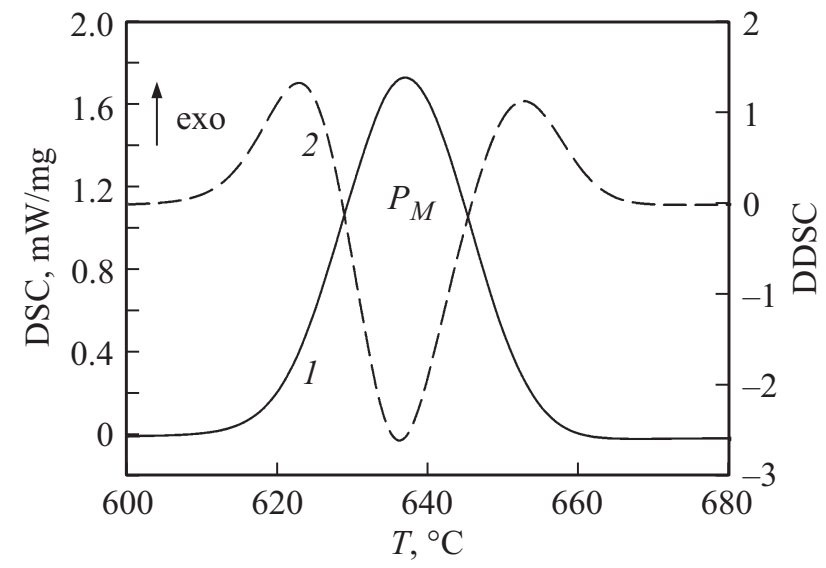

Рис. 2. Зависимость сигнала DSC (1) и DDDSC (2) от температуры при кристаллизации алюминия.

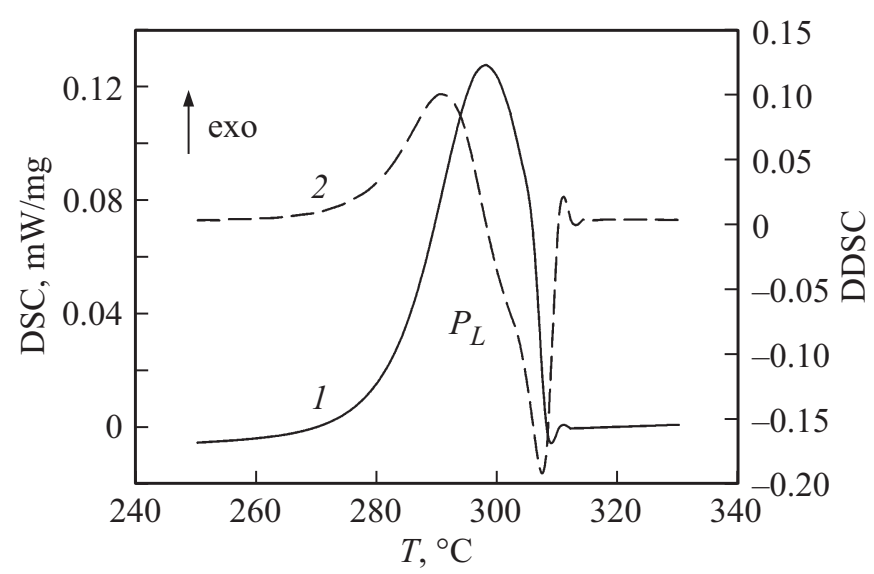

Рис. 3. Зависимость сигнала DSC (1) и DDSC (2) от температуры при кристаллизации сплава $\mathrm{Bi}-\mathrm{Sn}(5 \% \mathrm{Sn})$.

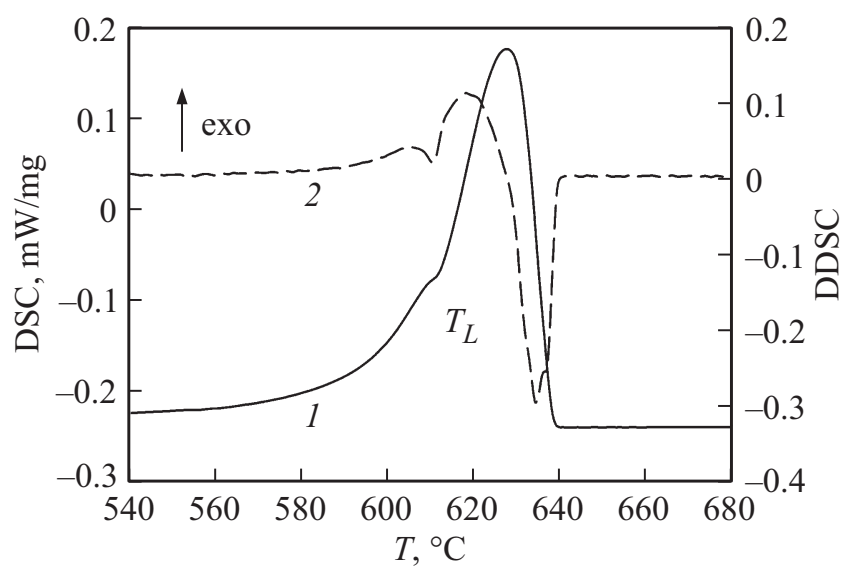

Рис. 4. Зависимость сигнала DSC (1) и DDSC (2) от температуры при кристаллизации сплава $\mathrm{Al}-\mathrm{Cu}(4 \% \mathrm{Cu})$.

нонвариантным равновесием на диаграммах состояния двухкомпонентных сплавов.

Совсем иная ситуация наблюдается при кристаллизации двухкомпонентных сплавов первого типа (см. рис. 3 и 4). По достижению линии ликвидуса 
наблюдается скачок выделения тепла. Об этом же свидетельствует вид первой производной сигнала DSC в этой области температур. Если использовать правило отрезков для определения количества новой фазы, образующейся непосредственно при переходе линии ликвидуса, то возникающее количество кристаллизующейся фазы относительно невелико. С этой точки зрения ее появление не должно было бы сопровождаться таким заметным скачком выделения тепла, поскольку поток тепла определяется теплотой превращения и объемной долей образования новой фазы (см., например, [5]).

Более того, если с применением правила отрезков построить зависимость объемной доли кристаллизующейся фазы от температуры охлаждения в межкритическом интервале температур (скорость охлаждения постоянна), то приведенная объемная скорость превращения должна проходить через максимум в интервале температур между линиями ликвидуса и солидуса. Данные DSC этого не подтверждают.

В принципе, такая же картина наблюдается и при кристаллизации сплавов доэвтектического состава, претерпевающих при охлаждении из жидкого состояния эвтектическую реакцию (II тип). Пример DSC зависимости для этого случая представлен на рис. 5.

Характер изменения сигнала DSC при эвтектической реакции (пик $\left.P_{e}\right)$ в этом сплаве отличается от данных, представленных на рис. 1. Наблюдается заметная асимметрия пика на DSC зависимости и фиксируется тенденция к ускорению выделения тепла по достижению линии эвтектической реакции. Можно предположить, что наличие в жидкости одной из фаз, выделившейся при переходе линии ликвидуса, создает благоприятные условия для начала кристаллизации другой фазы, также участвующей в эвтектической реакции.

Во многом похожее поведение сигнала DSC наблюдается и при охлаждении сплава заэвтектического состава (см. рис. 6). Здесь также по достижению линии ликвидуса фиксируется скачок выделения тепла и некоторая деформация сигнала DSC в ходе эвтектической реакции. Опять же можно допустить, что и здесь такая особенность связана с активизацией выделения другой фазы, участвующей в образовании эвтектики.

Отмеченные закономерности наблюдаются и при кристаллизации других двухкомпонентных сплавов: $\mathrm{Pb}-\mathrm{Sn}$, $\mathrm{Pb}-\mathrm{Bi}, \mathrm{Bi}-\mathrm{Sn}, \mathrm{Al}-\mathrm{Cu}$ (см. [12-14]). Следует отметить, что такие же закономерности в изменении сигнала DSC и DDSC при кристаллизации сплавов системы $\mathrm{Al}-\mathrm{Si}$ получены и в работе [12], но эти особенности там вообще не обсуждаются.

Можно предложить следующее объяснение установленным закономерностям. Для того чтобы из жидкости началось выделение кристаллов, состав которых заметно отличается от среднего состава компонентов в жидком состоянии, в этой жидкости при приближении к линии ликвидуса должно произойти расслоение на области с относительно низкой и относительно высокой, близкой к составу возникающих кристаллов, концентрацией одного

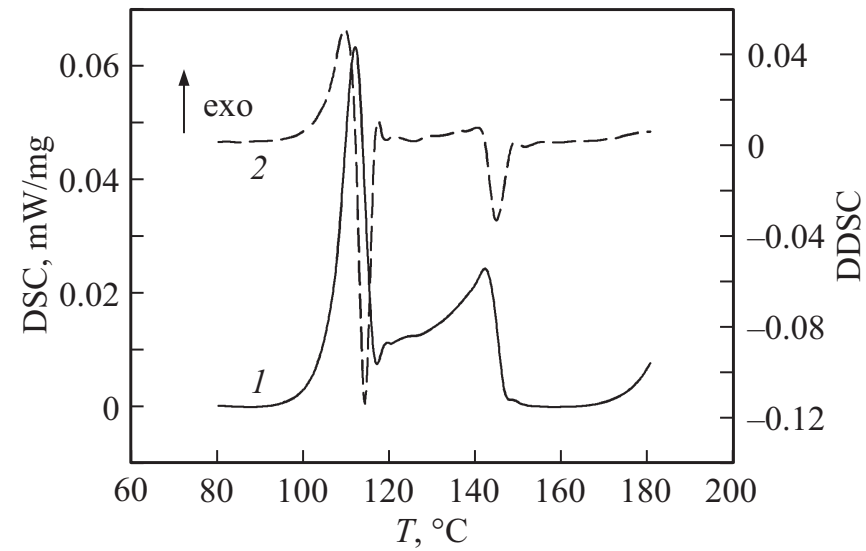

Рис. 5. Зависимость сигнала DSC (1) и DDSC (2) от температуры при кристаллизации сплава $\mathrm{Bi}-\mathrm{Sn}(20 \% \mathrm{Sn})$.

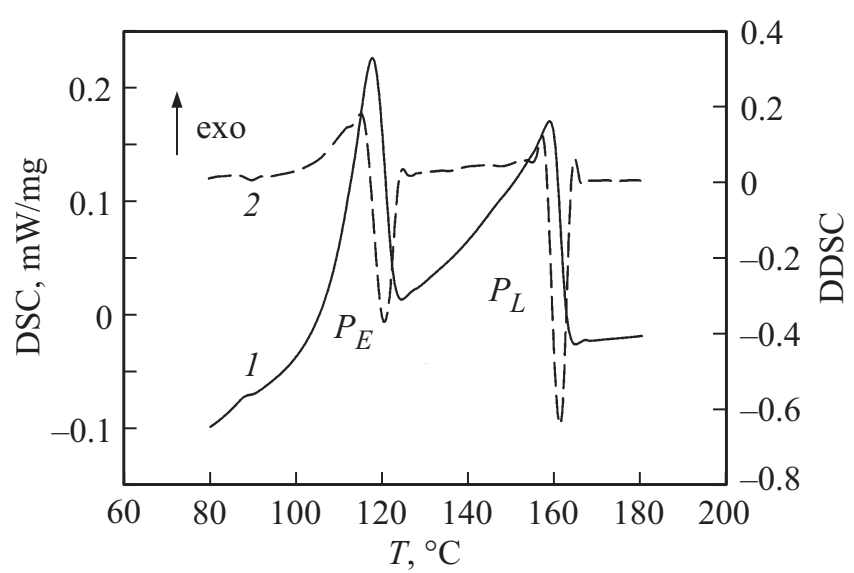

Рис. 6. Зависимость сигнала DSC (1) и DDSC (2) от температуры при кристаллизации сплава $\mathrm{Bi}-\mathrm{Sn}(60 \% \mathrm{Sn})$.

из компонентов. Происходит нечто близкое к спинодальному распаду пересыщенных твердых растворов [15].

При переходе линии ликвидуса в таких уже „подготовленных“ к возникновению новой фазы объемах спонтанно происходит образование большого числа центров кристаллизации. В этот процесс вовлекается заметная часть кристаллизующейся жидкости. В совокупности, как предполагается, это приводит к интенсивному тепловыделению при переходе линии ликвидуса.

Пик $P_{L}$ на DSC кривых охлаждения сплавов I типа можно представить как наложение двух перекрывающих друг друга подпика (см. рис. 7). Высокотемпературный подпик, имеющий справа резкий фронт, связан, повидимому, с возникновением новых кристаллов. Тогда как второй (низкотемпературный), более симметричный, обусловлен ростом кристаллов. Данное обстоятельство можно считать некоторым подтверждением развиваемых в работе представлений об особенности процесса кристаллизации в двухкомпонентных сплавах с диаграммами состояния I-III рода. 


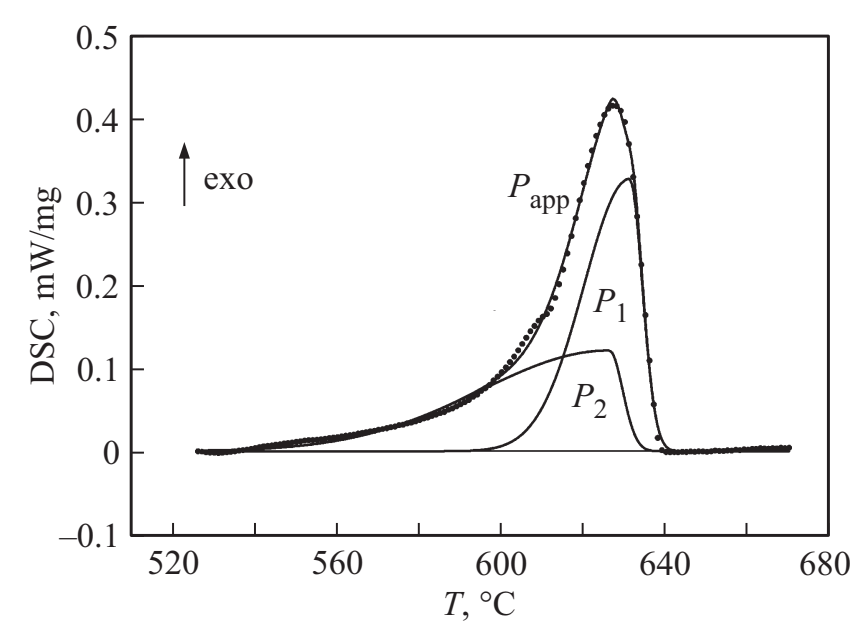

Pис. 7. Строение экзотермического пика на рис. 4. Точки экспериментальные данные; $P_{a p p}-$ результат аппроксимации; $P_{1}$ и $P_{2}-$ подпики.

В подтверждение высказанного предположения можно привести следующие соображения.

Согласно принятым представлениям максимальная скорость возникновения центров кристаллизации и скорость роста кристаллов наблюдается при температурах, значительно более низких, чем температура начала кристаллизации [3-5]. Это считается справедливым для однокомпонентных жидкостей.

В контексте выше сказанного, представляется целесообразным остановиться на попытках теоретического определения скоростей зарождения центров кристаллизации.

Одна из них связана с работами Френкеля (см. $[1,2])$, согласно которым скорость зарождения центров кристаллизации можно представить произведением двух экспоненциальных функций, зависящих от величины переохлаждения $\Delta T$. В этом случае максимум скорости зарождения центров кристаллизации лежит при $1 / 2 T_{s}<\Delta T<2 / 3 T_{s}$, где $T_{s}-$ температура плавления в $K$.

Другой подход предложен Чалмерсом [3,4]. Предполагается, что число зародышей, состоящих из $n_{i}$ атомов, определяется из следующего выражения

$$
n_{i}=n \exp [-\Delta G / k t]
$$

где $n$ - общее число атомов в единице объема, $\Delta G-$ изменение свободной энергии при образовании зародыша кристаллизации.

Если предположить, что каждый зародыш критического размера вырастает в кристалл, и таким образом его можно исключить из статистического распределения кластеров по размерам, то соответствующая скорость образования зародышей определяется величиной, с которой более мелкие кластеры достигают критического размера.
Для скорости образования зародышей им предложено следующее выражение:

$$
v=n_{s}^{*} \varepsilon v_{L} n \exp \left[-\left(\Delta G_{d}^{*}+\Delta G^{*}\right) / k T\right],
$$

где $n_{s}^{*} \approx 100$ число атомов жидкости, контактирующих с поверхностью критического зародыша, $\varepsilon \sim 1 / 6-$ вероятность перескока атома в заданном направлении, $v_{L} \sim 10^{13}$ - частота атомных колебаний, $\Delta G_{d}^{*} \sim k T-$ свободная энергия активации процессов диффузии в расплаве.

Если это уравнение представить графически, то можно увидеть, что при переохлаждении $\sim 0.2 T_{s}$ наблюдается быстрое возрастание скорости образования зародышей, причем она настолько чувствительна к величине экспоненциального члена в этом уравнении, что изменение предэкспоненциального множителя на несколько порядков, не оказывает существенного влияния на величину переохлаждения.

Результаты применения метода диспергирования образцов кристаллизующейся жидкости на капли микроскопического размера были обобщены Торнбаллом [3], и для большинства жидкостей $\Delta T_{\max }>0.15 T_{s}$, для ГЦК и ОЦК-металлов $\Delta T_{\max } \sim 0.18 T_{s}$. Это достаточно близко к теоретическому анализу ситуации. Обзоры по затвердеванию жидкостей, опубликованные в последнее время (см. $[4,5])$ мало что изменили в представлениях о скорости зарождения центров кристаллизации.

Принципиальной особенностью исследований по определению скорости образования центров кристаллизации и скорости их последующего роста является то, что они предполагают проведение изотермических экспериментов при заданной величине переохлаждения $\Delta T$. Но и в этих монографиях практически не рассмотрены процессы при кристаллизации двухкомпонентных сплавов. Приведенные же данные по кристаллизации эвтектических расплавов, касаются не скорости зарождения новых кристаллов, а скорости роста ячеек с чередующимися выделениями двух одновременно кристаллизующихся фаз (см., например, [1-5]).

Совершенно иная ситуация реализуется при непрерывном охлаждении расплавов. При достижении температуры кристаллизации однокомпонентного сплава согласно правилу фаз наступает нонвариантное равновесие. Наблюдается остановка на кривых, характеризующих изменение температуры во времени при охлаждении жидкой фазы. По нашим данным максимальная скорость кристаллизации при охлаждении однокомпонентных жидкостей чистых металлов характеризуется при скорости охлаждении $10 \mathrm{~K} / \mathrm{min}$. следующим рядом значений: $\mathrm{Sn}-0.021 T_{s}^{*}, \mathrm{Bi}-0.015 T_{s}^{*}, \mathrm{~Pb}-0.021 T_{s}^{*}$, $\mathrm{Zn}-0.026 T_{s}^{*}, \mathrm{Al}-0.015 T_{s}^{*} \mathrm{Ag}-0.012 T_{s}^{*}, \mathrm{Au}-$ $0.011 T_{s}^{*}, \mathrm{Cu}-0.013 T_{s}^{*}$. Здесь $T_{s}^{*}-$ температура плавления, определенная по данным DSC. В работе [5] она дается как $0.15 T_{s}$. 
Столь малая величина переохлаждения позволяет высказать предположение, что скорость зарождения центров кристаллизации не является лимитирующим фактором при кристаллизации однокомпонентных жидкостей. Если это предположение справедливо, то отсюда следует, что при приближении к температуре кристаллизации в жидкой фазе возникают многочисленные кластеры, расположение атомов в которых близко к пространственному расположению атомов в кристалле.

Согласно Чалмерсу [3-5], такой кластер должен состоять приблизительно из 100 атомов. Теория Френкеля $[1,2]$ дает размер критического зародыша на порядки больше ( $10^{4}$ атомов). Однако не только теоретические расчеты, но и некоторые эксперименты показывают (см. [1-5]), что ближний порядок в расположении атомов жидкости согласуется с их расположением в кристаллической фазе. Здесь же можно упомянуть и об идее существования сиботаксических групп атомов, расположение которых близки к их расположению в кристалле. Эти представления в определенной степени интегрированы в работе [5].

На затруднение процесса кристаллизации в тех случаях, когда состав кристаллов отличается от состава жидкой фазы, в свое время обратил внимание Пуассон $[1,16]$. Рассмотренная выше теория кристаллизации достаточно полно охватывает лишь те процессы, в результате которых состав твердой фазы совпадает с составом затвердевающей жидкости: кристаллизацию расплавленных чистых металлов и гомогенных химических соединений. Только в этих случаях вероятность образования равновесного кристаллического зародыша определяется одной лишь необходимостью создания поверхности раздела между твердой и жидкой фазами и равна вероятности энергетической флюктуации.

Если среднее число молеул или атомов кристаллизующегося вещества, приходящегося на объем жидкости, равный объему равновесного зародыша, обозначить через $\varepsilon$, а число тех же молекул (атомов) в закристаллизовавшемся зародыше через $j$, то вероятность существования соответствующей флюктуации выразится уравнением Пуассона [16]

$$
P_{s}^{j}=\frac{\varepsilon^{j} \cdot e^{-\varepsilon}}{j !} .
$$

Таким образом, не только при кристаллизации однокомпонентной жидкости, но в первую очередь при кристаллизации двухкомпонентных расплавов вблизи температуры кристаллизации (ликвидуса) происходит расслоение жидкой фазы на на огромное число кластеров, структура которых и соотношение компонентов в которых близки к таковым для возникающей кристаллической фазы. Как следствие, при переходе линии ликвидуса в значительном объеме жидкой фазы возникают многочисленные кристаллы. Спонтанное появление большого числа таких кристаллов приводит к наблюдаемым калориметрическим эффектам. Правило отрезков указывает на появление весьма незначительного количества новой фазы сразу же после перехода линии ликвидуса. Поэтому его применение не объясняет установленные в работе закономерности.

Для сплавов не эвтектического состава по данным термического анализа (DTA) можно определить расположение линий ликвидуса и солидуса, что и осуществляется в большинстве случаев при построении диаграмм состояния двухкомпонентных сплавов. Однако DTA методы не дают какой-либо информации о процессах, происходящих в интервале температур между линиями ликвидуса и солидуса. Проведение же структурных исследований in situ при повышенных и, тем более, высоких температурах, достаточно проблематично.

В этом плане методы DSC высокого разрешения, как показано в данной работе, позволяют дополнить существующие представления о закономерностях кристаллизации двухкомпонентных сплавов различного состава на диаграммах состояния I-III рода.

Надо полагать, что обнаруженные в данной работе закономерности, то есть скачок тепловыделения, могут иметь место и при распаде пересышенных твердых растворов. Некоторые экспериментальные доказательства этого получены в работе [13].

\section{4. Заключение}

Для кристаллизации чистых металлов определены величины переохлаждения, отвечающие максимальной скорости выделения теплоты превращения. Величина переохлаждения меньше $0.02 T_{s}^{*}$. Это может быть свидетельством того, что максимальные скорости зарождения и роста кристаллов наблюдаются при малых величинах переохлаждения вблизи температуры кристаллизации.

Показано, что при кристаллизации сплавов на базе систем $\mathrm{Pb}-\mathrm{Sn}, \mathrm{Pb}-\mathrm{Bi}, \mathrm{Bi}-\mathrm{Sn}, \mathrm{Al}$ - вблизи линии ликвидуса резко возрастает скорость выделения тепла кристаллизации, которая затухает по мере исчерпания жидкой фазы.

Высказано предположение, что начало кристаллизации при приближении к линии ликвидуса двухкомпонентных сплавов, связано с образованием в жидкости обогащенных ведущим кристаллизацию компонентом локальных микрообъемов, кластеров, появление которых предшествует началу процессов образования и роста кристаллов новой фазы. Скачкообразный характер развития кристаллизационного процесса сопровождается активным выделением тепла сразу же после перехода линии ликвидуса.

\section{Список литературы}

[1] Я.С. Уманский, Б.И. Финкельштейн, М.Е. Блантер. Физическое металловедение. Металлургиздат, М. (1958). 721 с.

[2] Я.С. Уманский, Ю.А. Скаков. Физика металлов. Атомиздат, M. (1978). $352 \mathrm{c}$. 
[3] H. Biloni, W.J. Boetinger. In Physical Metallurgy, 4th ed. / Ed. R.W. Cahn, P. Haasen. North-Holland, Amsterdam (1996). P. 669.

[4] M.E. Glicksman. Principles of Solidification: An Introduction to Modern Casting and Crystal Growth Concepts. Springer (2011). 530 p.

[5] W. Kurz, D.J. Fisher. Fundamentals of Solidification. 4th ed. Trans. Tech. Publication, Ltd. Switzerland. (1998). 305 p.

[6] Н.С. Курнаков. Введение в физико-химический анализ. 4-е изд. Изд-во АН СССР. М.-Л. (1940). 562 с.

[7] К. Судзуки, Х. Фудзимори, К. Хасимото. Аморфные металлы. Металлургия. М. (1987). 328 с.

[8] B. Cantor. J. Thermal Anal. 42, 4, 647 (1994).

[9] Л. В. Спивак. А.В. Шеляков. Изв. РАН. Физика 73, 9, 1337 (2008).

[10] L. Heusler, W. Schneider. J. Light Met. 2, 17 (2002).

[11] J. Piątkowski, V. Przeliorz, V. Szymszal. Archives Foundry Eng. 17, 2, 207 (2017).

[12] J. Piạtkowski, B. Gajdzik. MetaBK 52, 4, 469. (2013).

[13] Л.В. Спивак, Н.Е. Щепина Фундам. пробл. соврем. материаловедения 11, 3, 376 (2014).

[14] Л.В. Спивак. Вестн. Пермского ун-та. Сер. физ. 1, $26,9$. (2014).

[15] А.В. Скрипов, В.П. Скрипов. УФН 128, 2, 193 (1979).

[16] Е.С. Вентцель, Л.А. Овчаров. Теория вероятностей и ее инженерные приложения. 2-е изд. Высш. шк., М. (2000). $480 \mathrm{c}$.

Редактор Т.Н. Василевская 